\title{
Surgical Aspects of a Lower Face, Mandible, and Tongue Allotransplantation
}

\author{
Pedro C. Cavadas, M.D., Ph.D. ${ }^{1}$ Javier Ibáñez, M.D. ${ }^{1}$ Alessandro Thione, M.D., Ph.D. ${ }^{1}$ \\ ${ }^{1}$ Department of Reconstructive Surgery, Clinica Cavadas, Valencia, \\ Spain \\ Address for correspondence and reprint requests Pedro C. Cavadas, \\ M.D., Ph.D., Clinica Cavadas, Paseo Facultades 1, Valencia 46021, \\ Spain (e-mail: pcavadas@clinicacavadas.es).
}

J Reconstr Microsurg 2012;28:43-48.
Abstract Keywords
- face transplantation
- tongue transplantation
- malignancy
- radionecrosis
- HIV
- vascularized composite allotransplantation

\begin{abstract}
Face transplantation is a novel treatment for the reconstruction of massive facial defects. To date 13 cases have been performed. The technical aspects of a composite lower face transplantation including the tongue, floor of the mouth, and most of the mandible are detailed. The transplantation was performed in August 2009 in an HIVpositive, postoncologic patient. A preparatory surgery for nerve identification was performed. Facial composite tissue was procured after cardiac cessation. Revascularization was performed to the right subclavian artery with an internal shunt between the internal carotid arteries. At 16 months posttransplantation the patient is swallowing, without evidence of malignancy recurrence or HIV replication.
\end{abstract}

Vascularized composite allotransplantation (VCA) is progressively being used in reconstructive surgery. Due to improvements in immunosuppression and clinical management of patients with solid organ transplantations, VCA has been used in the reconstruction of upper extremity amputations, with good short- and midterm results, and limited morbidity. ${ }^{1}$ Attention has been directed recently to the reconstruction of massive facial defects with facial transplantation. Although a VCA including the scalp and the ears was reported in an advanced melanoma patient in 2005 with conceivably poor outcome, ${ }^{2}$ the first genuine facial transplantation was performed in France in 2006. ${ }^{3,4}$ At the time of this writing, 13 cases have been performed in 4 countries, 7 in France, 3 in Spain, 2 in the United States, and 1 in China., 5, 7,8,9,10 The etiology of the facial defects was traumatic in most cases, with two cases of extensive facial neurofibromatosis. The transplanted facial segments have included mainly the lips, nose, varying segments of maxilla and/or anterior mandible, and other aesthetic units of the face. Recently a case that included the four eyelids has been reported. ${ }^{11}$ A case of tongue transplantation to treat longue necrosis as a result of surgical treatment of an advanced malignancy of the

received

February 13, 2011

accepted after revision

May 3, 2011

published online

July 21, 2011

floor of the mouth was reported in 2008 with short-patient survival. $^{12}$

The purpose of this article is to report on the technical aspects of a lower face composite allotransplantation including the tongue, floor of the mouth, and most of the mandible performed in Spain in August 2009. The facial defect was secondary to complications of radiotherapy for a squamous cell carcinoma (SCC) of the floor of the mouth in an HIVpositive patient. To the best of the authors' knowledge, this is the first case of a facial transplantation including the tongue, and the first performed in an HIV-positive or postoncologic patient.

\section{Patient and Methods}

\section{The Recipient Patient}

The recipient patient was an HIV-positive, 42-year-old male on highly active antiretroviral therapy (HAART) (ritonavir, darunavir, raltegravir, etravirine, and enfuvirtide) with CD4 counts over $400 / \mathrm{mL}$ and negative viral load. The facial defect included the lower lip, tongue, floor of the mouth, and the body of the mandible, with septic remnants of mandibular

Copyright (c 2012 by Thieme Medical Publishers, Inc., 333 Seventh Avenue, New York, NY 10001, USA.

Tel: +1(212) 584-4662.
DOI http://dx.doi.org/ 10.1055/s-0031-1284236. ISSN 0743-684X. 


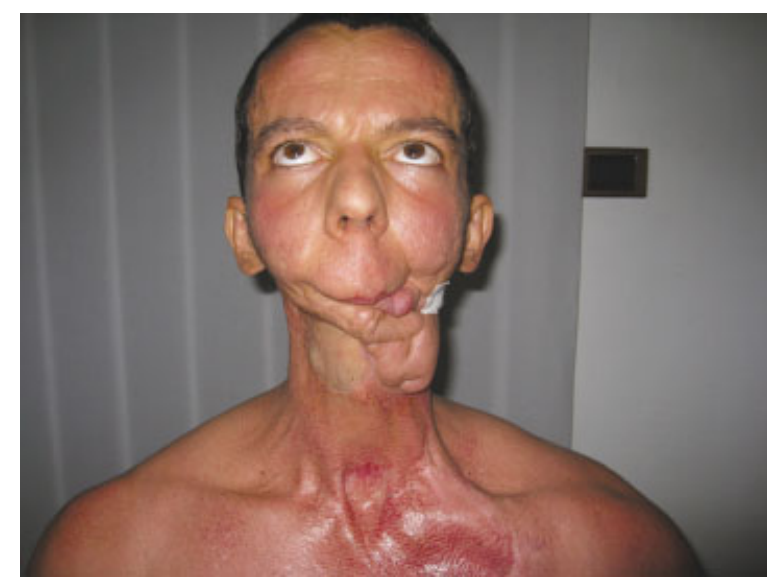

Figure 1 The composite lower face defect. There was massive scarring and soft tissue collapse, and septic fistulae.

bone bilaterally. The facial defect was secondary to complications of mandibular radionecrosis after chemoradiotherapy for a SCC of the tongue (T3NOMO) 11 years before. Multiple attempts at surgical reconstruction (including five free and one pedicled flap performed elsewhere) compounded the defect (-Fig. 1). The neck was massively scarred and rigid, with virtually absent recipient vessels. There was a radiation injury to the larynx, with vocal cord palsy in paramedian position, and a bony ankylosis of the left temporomandibular joint (TMJ).

The patient was fully informed of the limited possibilities of further autologous reconstructions, and the limited experience in human facial transplantation at that time. The fact that VCA had never been performed before in HIV-positive patients, the limited information available about tongue transplantation, the risks of transplantation after malignancy, and the results of solid organ transplantations in HIV-positive recipients pertinent to his case were fully explained to the patient. After approval by an ethical committee, a composite lower facial-mandible-tongue VCA was proposed to the patient. IRB and National Organization for Organ Transplantation (Organización Nacional de Trasplantes, ONT) approvals were also obtained.

\section{Preparatory Surgery}

An elective surgery was performed ( 3 months before transplantation and 1 month before the patient entered the waiting list) to debride the septic bone remnants of the mandible, and to identify and tag the recipient nerves without time constraints. Hypoglossal, lingual, inferior alveolar, and lower divisions of the facial nerves were dissected bilaterally off the thick scar and tagged with colored silicone loops. The infected remnants of previous attempts at osseous reconstruction were debrided, and the remnants of the upper denture were removed. The bone defect included the body of the mandible and the left hemimandible. The left condyle was drilled up to the temporal bone, and a solid spacer of polymethyl-methacrylate with gentamycin was inserted in the condylar region to avoid soft-tissue collapse, and allow space for the allogeneic condyle.

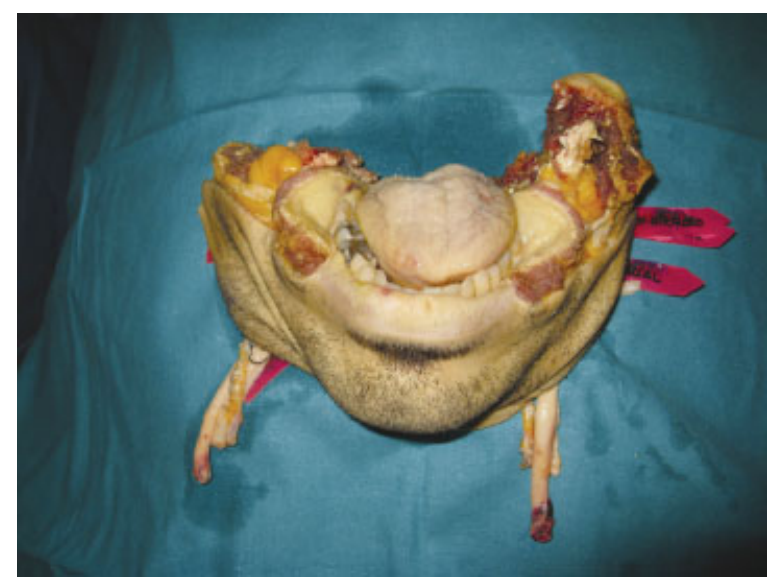

Figure 2 The VCA harvested from the donor, including the lower face, tongue, floor of the mouth, salivary glands, and three-fourths of the mandible.

\section{Transplantation Surgery}

Face transplantation was performed on August 2009, by a single surgical team, with sequential dissection on the recipient, procurement of the VCA from the donor, and insettingrevascularization. The subclavian artery, internal and external jugular veins on the right side, and the left external jugular vein on the left side were dissected as recipient vessels. The previously tagged recipient nerves were identified and the methacrylate condylar spacer was removed. The scarred tissue of the lower third of the face was removed and the scarred mucosa of the oropharynx was released.

Facial VCA procurement was performed immediately after cardiac cessation, from a fully HLA-mismatched multiorgan brain-dead donor. The aorta was cross- clamped distal to the left subclavian take-off to allow the in situ perfusion of both carotid arteries with cold Celsior preservation solution immediately before heart procurement. The facial VCA included the skin from the oral commissures to the earlobes, and down to the upper neck, the mandible from the right angle to the left condyle, the tongue, the floor of the mouth, the hyoid bone, suprahyoid muscles, and the six major salivary glands ( - Figs. 2 and 3). The hypoglossal, lingual, inferior alveolar, and lower division of the facial nerves were dissected up to the skull base. The left mylohyoid nerve was identified on the inferior alveolar nerve. The right external carotid artery was dissected, the upper thyroideal, ascending pharyngeal, and posterior auricular arteries were ligated and divided, and the lingual and facial arteries were included. In the left side, the internal maxillary artery and its inferior alveolar branch were included, along with the lingual and facial arteries. The common and internal carotid arteries were dissected and included in the VCA bilaterally. External jugular, thyrolingual, and internal jugular veins were also included bilaterally. VCA harvesting took 3.5 hours (cold ischemia). An end-to-end anastomosis was performed between the distal ends of both internal carotid arteries at the side table to enhance contralateral arterial inflow (-Fig. $\mathbf{3}$ ).

After minimal transportation time, revascularization of the VCA was performed. End-to-end anastomosis of the right 


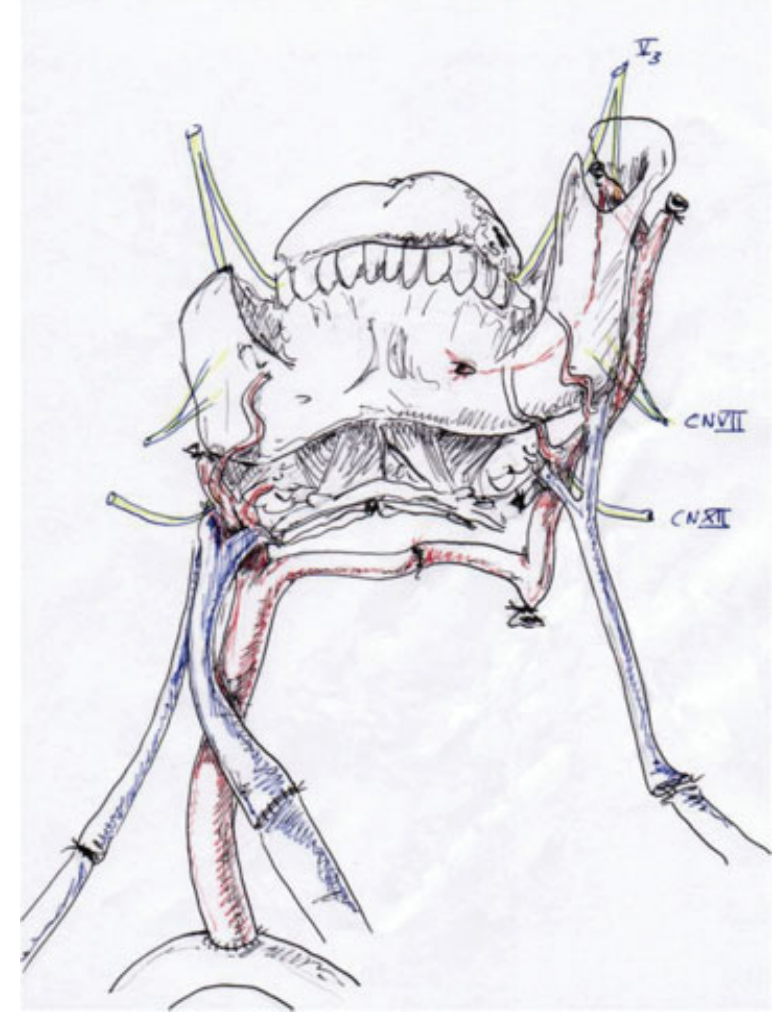

Figure 3 Schematic drawing of the VCA and vascular connections. CN VII, facial nerve; CN XII, hypoglossal nerve; V3, third division of the trigeminal nerve.

internal jugular veins and end-to-side anastomosis between the right subclavian and the right common carotid arteries were performed before the insetting. Hemostasis of the flap was performed and the left external jugular vein was anastomosed to the homonymous vessel. The oral and oropharyngeal mucosae were sutured; the left hypoglossal, lingual, and inferior alveolar nerves were coapted trying to include the left mylohyoid nerve in an attempt at reinnervation of the suprahyoid muscles. The left mandibular condyle was fixed with a heavy resorbable suture to the soft tissues of the temporomandibular fossa, and the left lower facial nerve was coapted. On the right side, the lingual and hypoglossal nerves were coapted (a short nerve graft was used for the hypoglossal), the mandible was plated with a single 2-mm plate, the inferior alveolar nerve was coapted, a short graft was used for lower facial nerve repair, and the skin was closed.

\section{Results}

\section{Clinical Evolution}

The immunosuppressive treatment included induction with anti-CD25 basiliximab and standard triple therapy with tacrolimus, mycophenolate mofetil, and corticosteroids. Calcineurin inhibitor dosing was modified according to previously described interactions with antiretroviral

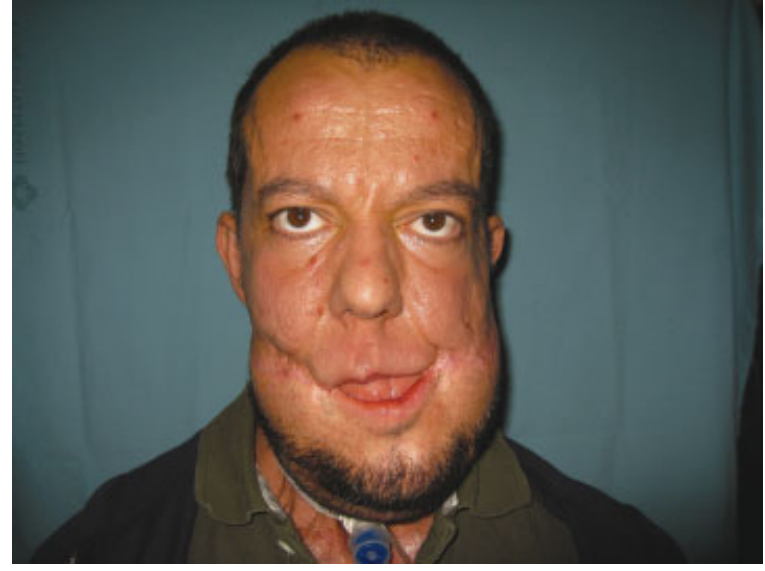

Figure 4 Result at 16 months posttransplantation.

drugs. ${ }^{13}$ Although the details of acute rejection episodes, clinical course, and functional results are beyond the scope of the present study, at 16 months posttransplant the patient is rejection-free, with no evidence of HIV replication or malignancy recurrence, is swallowing, and starting phonation rehabilitation (-Fig 4). Mandible excursion is $10 \mathrm{~mm}$ probably due to previous injury to the right TMJ. Two acute rejection episodes occurred on postoperative days 14 and 350 (Banff histological grade III) ${ }^{14}$ and responded to intravenous methylprednisolone $500 \mathrm{mg} / 24 \mathrm{~h}$ for 5 days. At postoperative month 11 a pseudosarcomatous spindle-cell postsurgical nodule was removed from the base of the tongue through a sagittal mandible-splitting approach. Although the histological diagnosis of pseudotumor was firm, tacrolimus was switched to sirolimus. The nodule has not recurred.

\section{Discussion}

Autologous reconstruction of massive facial defects, especially those involving the perioral and periocular regions yields modest results at best. Facial transplantation has emerged as a novel treatment for these unfortunate patients. With 13 cases performed by 8 different teams at the time of this writing, facial transplantation should still be considered an experimental procedure. Issues such as longterm results, immunological singularities of the face, and the role of bone marrow in facial transplantation are largely unanswered questions. From a medical standpoint, neither the oncological precedents nor the HIV status of the patient contraindicated per se the transplantation. ${ }^{15}$ Solid organ transplantation has been successfully reported in HIV-positive patients with negative viral load and CD4 counts over 200 cells $/ \mathrm{mL}$. A disease-free interval of 5 years after malignancy is commonly considered safe before solid organ transplantation. Although VCA has not been reported before in either category of oncologic and HIV-positive patients, it is the opinion of the authors that in severe cases the risk-benefit ratio is favorable in these medically complex recipients. 
Pseudosarcomatous spindle-cell nodule is a benign reparative pseudotumor described mainly in the bladder and less frequently in the pharynx, after surgical insults. Pathological diagnosis is challenging as it can be easily mistaken for a highgrade malignancy. Complete surgical resection is adequate curative treatment. ${ }^{16}$

In contradistinction to hand transplantation, facial defects and facial transplants are remarkably heterogeneous from an anatomical point of view. Surgical considerations are thus especially pertinent to facial transplantation. Some details of this case deserve comment. Facial VCA procurement from the donor after cardiac cessation and exsanguination makes dissection more expeditious, and avoids the significant bleeding associated and any potential interference with the procurement of other vital organs. On the other hand, it prolongs ischemia time, it needs special training to identify empty vessels, and effective hemostasis is more difficult to achieve. The in situ perfusion with cold preservation solution before VCA dissection probably makes this increase in ischemia time less relevant. ${ }^{17}$ Although it has been demonstrated that increased ischemia time is detrimental to muscle function ${ }^{18}$ and increases acute rejection episodes, ${ }^{16}$ the global benefits of dry harvesting the face may outweigh the drawbacks. To compensate for this extra ischemia time and to allow for effective hemostasis, the revascularization was performed before the insetting. The bleeding was not profuse, but progressive swelling of the flap made insetting of the right side very difficult, and dictated the use of nerve grafts for the right facial and hypoglossal nerves. Probably the insetting should have been done before revascularization, at the price of 2 more hours of ischemia time.

Anatomically, this case had some unique features. The incorporation of the tongue and the left hemimandible dictated the inclusion of bilateral lingual arteries, lingual and hypoglossal nerves, and the left internal maxillary artery and its inferior alveolar branch (-Fig. 2). Musculoperiosteal blood supply can nourish segments of the mandible, but the endosteal system from the inferior alveolar artery is necessary for hemimandibular or larger segments. ${ }^{19}$ The massive scarring of the neck posed significant difficulties to this case. The absence of recipient vessels in both sides of the neck dictated the use of the right subclavian artery as the sole recipient artery. Although the rich vascular network of the face allows contralateral flow across the midline with unilateral vascular supply, ${ }^{7,20,21}$ the end-to-end anastomosis of the internal carotid arteries is a simple and rapid surgical maneuver to increase this crossed circulation. Recipient nerve dissection and tagging was considered paramount for sensorimotor recovery and function of the VCA, and it was performed as an elective preparatory surgery to allow unrestricted operating time. This maneuver proved to be very helpful.

The inclusion of the maxillary bones and upper denture in the VCA, to obtain dental occlusion, was ruled out early during the planning of the case, on anatomical grounds. Because the upper dental arch was removed during the preparatory surgery, plans were made to fit an upper prosthetic denture according to that of the donor. Tongue swelling, the limited movement of the right TMJ, and the reduction of the mandible performed at the time of pseudotumor resection have delayed prosthodontic care. Likewise, the inclusion of the larynx in the composite VCA was initially considered because of the radiation injury and the vocal cord palsy, but the likelihood of satisfactory nerve regeneration after coaptation of the scarred superior laryngeal and recurrent nerves was considered poor and the idea abandoned.

In conclusion, a successful lower face composite VCA including the tongue and mandible was performed and the surgical details reported herein. To the best of the authors' knowledge this is the first case reported of this type of facial transplantations.

\section{Acknowledgments}

The authors want to acknowledge Marino Blanes, M.D., Luis Alfaro, M.D., Ph.D., and Luis Landin, M.D. for their contribution to the medical management of this patient.

\section{References}

1 Petruzzo P, Lanzetta M, Dubernard JM, et al. The international registry on hand and composite tissue transplantation. Transplantation 2010November 3 [Epub ahead of print]

2 Jiang HQ, Wang Y, Hu XB, Li YS, Li JS. Composite tissue allograft transplantation of cephalocervical skin flap and two ears. Plast Reconstr Surg 2005;115(3):31e-35e discussion 36e-37e

3 Devauchelle B, Badet L, Lengelé B, et al. First human face allograft: early report. Lancet 2006;368(9531):203-209

4 Dubernard JM, Lengelé B, Morelon E, et al. Outcomes 18 months after the first human partial face transplantation. N Engl J Med 2007;357(24):2451-2460

5 Lantieri L, Meningaud JP, Grimbert P, et al. Repair of the lower and middle parts of the face by composite tissue allotransplantation in a patient with massive plexiform neurofibroma: a 1-year followup study. Lancet 2008;372(9639):639-645

6 Guo S, Han Y, Zhang X, et al. Human facial allotransplantation: a 2-year follow-up study. Lancet 2008;372(9639):631-638

7 Pomahac B, Lengele B, Ridgway EB, et al. Vascular considerations in composite midfacial allotransplantation. Plast Reconstr Surg 2010;125(2):517-522

8 Gordon CR, Siemionow M, Papay F, et al. The world's experience with facial transplantation: what have we learned thus far? Ann Plast Surg 2009;63(5):572-578

9 Siemionow M, Papay F, Alam D, et al. Near-total human face transplantation for a severely disfigured patient in the USA. Lancet 2009;374(9685):203-209

10 Siemionow MZ, Papay F, Djohan R, et al. First U.S. near-total human face transplantation: a paradigm shift for massive complex injuries. Plast Reconstr Surg 2010;125(1):111-122

11 Meningaud JP, Benjoar MD, Hivelin M, et al. Procurement of total human face graft for allotransplantation: a preclinical study and the first clinical case. Plast Reconstr Surg 2010;126(4):11811190

12 Kermer C, Watzinger F, Oeckher M. Tongue transplantation: 10-month follow-up. Transplantation 2008;85(4):654-655

13 Danovitch GM. Immunosuppressive medications and protocols for kidney transplantation. In:Danovitch GMed.Handbook of Kidney Transplantation. 5th ed. PhiladelphiaLippincott Williams \& Wilkins201077-126 
14 Cendales LC, Kanitakis J, Schneeberger S, et al. The Banff 2007 working classification of skin-containing composite tissue allograft pathology. Am J Transplant 2008;8(7):1396-1400

15 Kasiske BL, Cangro CB, Hariharan S, et al. The evaluation of renal transplant candidates: clinical practice guidelines. Am J Transplant 2001;2(Suppl 1):5-95

16 Young RH. Tumor-like lesions of the urinary bladder. Mod Pathol 2009;22(Suppl 2):S37-S52

17 Henry ML, Pelletier RP. Renal allograft preservation. In:Weir MRed. Medical Management of Kidney Transplantation. Philadelphia Lippincott Williams and Wilkins2005365-371

18 Daigle JP, Kleinert JM. Major limb replantation in children. Microsurgery $1991 ; 12(3): 221-231$
19 Loukas M, Kinsella CR Jr, Kapos T, Tubbs RS, Ramachandra S Anatomical variation in arterial supply of the mandible with special regard to implant placement. Int J Oral Maxillofac Surg 2008;37(4):367-371

20 Banks ND, Hui-Chou HG, Tripathi S, et al. An anatomical study of external carotid artery vascular territories in face and midface flaps for transplantation. Plast Reconstr Surg 2009;123(6): 1677-1687

21 Wilhelmi BJ, Kang RH, Movassaghi K, Ganchi PA, Lee WP. First successful replantation of face and scalp with single-artery repair: model for face and scalp transplantation. Ann Plast Surg 2003;50 (5):535-540 